\title{
Human locomotion through a multiple obstacle environment: strategy changes as a result of visual field limitation
}

\author{
Sander E. M. Jansen • Alexander Toet • \\ Peter J. Werkhoven
}

Received: 27 January 2011/Accepted: 24 May 2011/Published online: 18 June 2011

(C) The Author(s) 2011. This article is published with open access at Springerlink.com

\begin{abstract}
This study investigated how human locomotion through an obstacle environment is influenced by visual field limitation. Participants were asked to walk at a comfortable pace to a target location while avoiding multiple vertical objects. During this task, they wore goggles restricting their visual field to small $\left(\mathrm{S}: 40^{\circ} \times 25^{\circ}\right)$, medium $\left(\mathrm{M}: 80^{\circ} \times 60^{\circ}\right)$, large $\left(\mathrm{L}: 115^{\circ} \times 90^{\circ}\right)$, or unlimited (U) visual field sizes. Full-body motion capture was used to extract for each trial the mean speed, pathlength, mean step width, magnitude of head rotation and head mean angular speed. The results show that compared with the U condition, the $\mathrm{M}$ and $\mathrm{L}$ conditions caused participants to select a wider path around the obstacles without slowing down or altering step width. However, the S condition did slow down the participants, and increased both their step width and path length. We conclude that only for the $\mathrm{S}$ condition, balancing problems were substantial enough to spend more energy associated with increased step width. In all cases, participants choose to optimize safety (collision avoidance) at the cost of spending more energy.
\end{abstract}

Keywords Locomotion - Steering - Obstacle avoidance · Visual field restriction $\cdot$ Head movement $\cdot$ Strategy changes

S. E. M. Jansen $(\bowtie) \cdot$ P. J. Werkhoven

Department of Information and Computing Sciences,

Utrecht University, PO Box 80.089, 3508TB Utrecht,

The Netherlands

e-mail: sanderj@cs.uu.nl

A. Toet

Perceptual and Cognitive Systems, TNO, PO Box 23,

3769ZG Soesterberg, The Netherlands

\section{Introduction}

Humans need to walk through structured environments without colliding with any obstacles or parts of that environment. In order to achieve this, there is a constant need for information concerning the surrounding space. Specifically, it is important to know the spatial relations between different parts of an environment (exteroception) to ensure that safe passage is possible. Furthermore, it is essential to have information concerning the position of the body in that environment (exproprioception). Both types of information need to be monitored during locomotion toward a goal. It is possible that distances between parts of the environment are misperceived initially and need to be corrected along the way. Moreover, the positioning of the body in the environment needs constant updating in order to predict and act upon potential future collisions.

Previous research has shown that obstacle size and position can be judged from a distance, while information concerning the position of the body in the environment is updated continuously during adaptive locomotion (Patla and Vickers 1997). In addition, Mohagheghi et al. (2004) showed that dynamic sampling (by means of head movement) prior to locomotion was sufficient to ensure safe obstacle crossing in the absence of vision in the approach phase. However, despite succeeding at the task, participants increased their safety margin around the obstacle.

Walking through a structured environment requires both steering of the body in a new travel direction and circumvention of obstacles situated in the travel path. Although related, these tasks are not the same. Circumvention requires a transient change in the center of mass (COM) while maintaining the underlying travel direction. Alternatively, during steering, the COM is guided in a new travel direction (Vallis and McFadyen 2003). Several 
studies on steering behavior reported a systematic sequence of body reorientations which is initiated by head yaw rotation (Patla et al. 1999a; Hollands et al. 2001). Next to visual cues, both vestibular and proprioceptive information (concerning the orientation of the head with respect to the torso) are important during navigational tasks. (Courtine and Schieppati 2003; Prévost et al. 2003). It is argued that the alignment of the head with the future direction of travel provides the CNS with an allocentric frame of reference (Hollands et al. 2002).

One important aspect of human locomotion is the need for energy conservation (Saunders 1953; Inman 1966; Zarrugh et al. 1974). Specifically, Donelan et al. (2004) propose that the metabolic cost of walking is largely determined by the work performed to redirect the COM and the accompanying medio-lateral foot placements that provide stabilization. They argue that humans prefer a step width that minimizes this cost. In addition, Bauby and Kuo (2000) proposed that during unobstructed walking straight ahead, this results in a step width of $0.12 L$ (where $L$ is leg length). Moreover, the increase in step width has been associated with postural instability (Gabell and Nayak 1984). Furthermore, Patla et al. (1999b) show that when participants are confronted with an undesirable landing area, the dominant choice for an alternative position is the one that requires least adjustment, thereby conserving energy.

Next to minimizing energy expenditure, there is also the concern of safety when negotiating obstacles. Much of the research done on safety during obstacle avoidance involves elderly people. It has been shown that compared with young adults, older subjects exhibit a more conservative gait pattern, characterized by reduced velocity and shorter step length (Menz et al. 2003; Paquette et al. 2008). Also, they employ a hip strategy during obstacle circumvention, as opposed to the foot placement strategy shown by younger adults (Paquette and Vallis 2010). Furthermore, Chapman and Hollands (2007) propose that older adults prone to falling prioritize the planning of future steps over the accurate execution of ongoing movement, which might actually cause accidents instead of preventing them.

Because elderly people are confronted with a multitude of risk factors influencing steering behavior (i.e., impaired vision, deficits to the muscoskeletal system, and impairment of the proprioceptive and vestibular systems), it is difficult to investigate the relation between a single impairing factor and the resulting steering behavior. Therefore, in this study, we focus solely on the influence of visual field limitation on locomotion through a multiobstacle environment.

Unrestricted, the human visual field is approximately $200^{\circ}$ wide and $135^{\circ}$ tall (Werner and Rossi 1991). However, this field size can be severely decreased by wearing devices such as head-mounted displays (HMDs) and nightvision goggles (NVGs) (Vargas-Martin 2002). Other causes for visual field limitation can be eye diseases such as retinitis pigmentosa (Turano et al. 1993) and glaucoma (Turano et al. 1999). Moreover, a common activity like carrying a large object will also cause partial occlusion of the lower visual field. It is therefore an important factor to investigate when studying human motor behavior.

The maintenance of postural balance is known to be impaired by visual field restriction (Paulus et al. 1984; Turano et al. 1993). Furthermore, observers tend to compensate for the reduction in their instantaneous visual field by making larger, but slower head movements (Wells and Venturino 1990; Szoboszlay et al. 1995). It is argued that the head movements are extended to counter the underestimation that otherwise results from an incomplete ground-surface integration (He et al. 2004; Wu et al. 2004; Creem-Regehr et al. 2005). Interestingly, Creem-Regehr et al. (2005) report giving explicit instructions to participants to 'look around'. This is based on observations that when wearing an HMD, people tend to limit their head movement if not explicitly instructed to do so. Moreover, with this instruction, participants did perform on par with the unrestricted viewing condition.

As a result of visual field restriction, optic flow is diminished. It has been argued that optic flow is used to control heading (Warren et al. 1991; Warren et al. 2001). However, others propose that visual guidance of locomotion is achieved not by optic flow, but by keeping targets and obstacles at fixed angles, or eccentricity, relative to the body (Rushton et al. 1998). In order to successfully steer through a structured environment, all of the above-mentioned subtasks (i.e., maintaining balance, distance estimation, and heading control) need to be performed correctly. Recently, a comprehensive review was written on the role of peripheral visual cues in the online guidance of locomotion (Marigold 2008).

In previous work, we showed that restriction of the visual field causes impairment on various obstacle avoidance tasks such as circumvention, ducking and crossing (Toet et al. 2008). Specifically, for local obstacle avoidance tasks, we observed that restriction of the vertical viewing angle causes greater performance degradation than that of the horizontal angle (Jansen et al. 2010). Subsequently, in a recent study, we investigated the effects of lower visual field restriction on obstacle crossing behavior (Jansen et al. 2011). It seems that compared with an unrestricted viewing condition, an intermediate vertical viewing angle $\left(40^{\circ}-90^{\circ}\right)$ causes participants to enlarge their safety margin when stepping over an obstacle by increasing toe clearance and step length. However, no change in speed was found. When confronted with a smaller viewing angle (i.e., $25^{\circ}$ ), perceived safety became even more compromised, resulting in 
a further enlargement of clearance as well as a decrease in speed. Consequently, it seems that for such an obstacle avoidance task, the size of the visual field has important consequences for the priority of behavioral strategies.

With the present study, we want to investigate whether visual field restriction affects steering in a similar manner as it does obstacle crossing. Using full-body motion capture, we examine the influence of four different visual field sizes on steering behavior when walking through an environment consisting of multiple obstacles. The results are discussed in terms of changes in priority between several optimization strategies. Specifically, we want to know whether speed preference has priority over energy conservation considerations as was found during the recently reported obstacle crossing task (Jansen et al. 2011). Furthermore, we are interested in the role of postural balance in this strategy shift. By investigating how step width is affected by visual field size, we gain insight into the postural instability that results from peripheral field loss. In addition, we want to know how head movement is altered as a consequence of visual field limitation. Previous work suggests that a decrease in visual field size causes an increase in the magnitude of head movement, but a decrease in its speed (Wells and Venturino 1990). This requires additional time which may be provided by a reduction in overall speed of movement. Finally, it is interesting to see if the performance plateau for medium to large visual field sizes found in previous studies (Toet et al. 2008; Jansen et al. 2011), can be replicated here.

Insight into human behavioral changes as a consequence of visual field limitation could benefit several application areas. First, it could be used to formulate requirements for the selection and development of field-of-view limiting devices, such as HMDs and NVGs when these are used during adaptive locomotion. Second, it contributes to the knowledge base on visually guided locomotion with a limited visual field. This could help in preventing falls in elderly as well as other people suffering from a limitation of their visual field.

\section{Methods \\ Participants}

The procedures of this study were approved by the TNO internal review board. Twelve paid participants gave informed consent and took part in the experiment. Three of them were excluded from analyses because of incomplete data sets (due to technical problems during the experiment). Eventually, nine participants were included in the analyses (four male) ranging in age from 21 to 59 years $(\mathrm{M}=33.6$; $\mathrm{SD}=15.3)$. All were free of any known neurological or orthopedic disorders, or any impediments to normal locomotion. As verified by self-report, all participants had normal or corrected-to-normal vision (by use of contact lenses).

Experimental materials

\section{Goggles}

Four separate pairs of safety goggles (type Bollé Targa; www.bolle-safety.com) were used to create each of the visual conditions: small $\left(\mathrm{S}: 40^{\circ} \times 25^{\circ}\right)$, medium $(\mathrm{M}$ : $\left.80^{\circ} \times 60^{\circ}\right)$, large $\left(\mathrm{L}: 115^{\circ} \times 90^{\circ}\right)$, and unrestricted (U). Part of the plastic lens was covered with duct tape in such a way that light from the specified visual field was permitted to enter the eye. Because of variations in facial bone structure, the exact viewing angles differed slightly per participant. However, the within-subjects design of the study ensures correct inference about the relation between visual field size and the performance measures. See the left panel of Fig. 1 for an example of the visual field restricting goggles.

\section{Motion capture}

Full-body motion was captured using the MVN inertial motion capture system by XSens (Roetenberg et al. 2009). Participants wore a Lycra suit equipped with 17 sensory modules, containing 3d gyroscopes, accelerometers, and magnetometers (see the right panel of Fig. 1 for a graphical representation). Furthermore, they wore their own comfortable walking shoes. Using the Xsens software, participants' full-body motion was recorded for each trial (update rate of $100 \mathrm{~Hz}$ ). A sensor fusion scheme calculated the position, velocity, acceleration, orientation, angular velocity, and angular acceleration of each body segment,

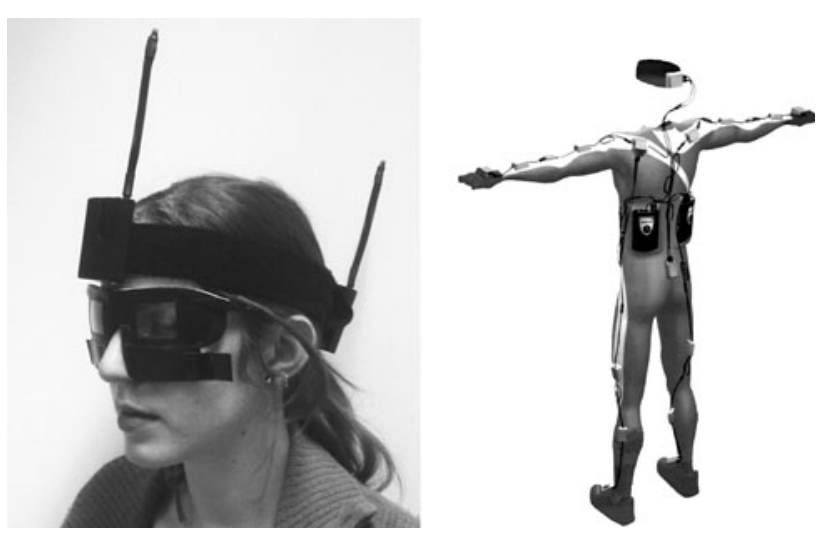

Fig. 1 Left image showing one of the visual field restricting goggles as well as the optical markers placed on a headband. Right MVN suit containing inertial sensory modules layout 
with respect to an earth-fixed reference coordinate system. The reader is referred to a paper by Roetenberg et al. (2009) for a more extensive description of this system.

Because of its inertial nature, the MVN system suffers from drift in absolute positioning. Therefore, an optical tracking system was employed as well (WorldViz 2005). This system returns the participant's exact position within a global world coordinate system by tracking optical markers (update rate of $60 \mathrm{~Hz}$ ). Participants wore a headband on which two markers were placed, one on the front and one on the back of the head. See the left panel of Fig. 1 for a photograph of the optical markers attached to the headband.

The following steps were undertaken to eliminate the absolute drift from the data: First, the inertial data were down-sampled to $60 \mathrm{~Hz}$ to match the optical data. Then, for each trial, we calculated the initial offset between the position of the head as given by MVN and the frontal optical marker. Second, for each frame, yaw and pitch orientation of the head was calculated from the $3 \mathrm{~d}$ positions of both optical markers. Third, from these orientations and offset, a 'new' head position could be generated. Finally, for each body segment in each frame, a new position was generated by taking the offset between that particular MVN segment and the original MVN head position for that frame and applying it to the new MVN head position.

\section{Environment}

The obstacle environment consisted of five open square wooden frames $(2,000 \mathrm{~mm} \mathrm{H} \times 2,000 \mathrm{~mm} \mathrm{~W} \times 15 \mathrm{~mm} \mathrm{D})$ placed one behind the other such that participants had to slalom from the start to goal positions in order to avoid collision with the obstacles. Distance between consecutive walls was $1 \mathrm{~m}$. See Fig. 2 for a schematic representation of the obstacle environment. The use of open wooden frames prevents occlusion of the markers, thereby enabling optical tracking.

\section{Design and procedures}

The four visual field conditions were randomized and performed four times each, resulting in 16 trials per participant. For each condition, the first trial was used to familiarize with each specific condition; only the last three trials were analyzed. Prior to execution of the experiment, participants gave informed consent and put on the Lycra sensor suit as well as the optical markers. After this, a calibration procedure was performed, in which the sensor to body alignment and body dimensions was determined for the inertial system. First, body height and foot size were measured. The other dimensions were obtained from
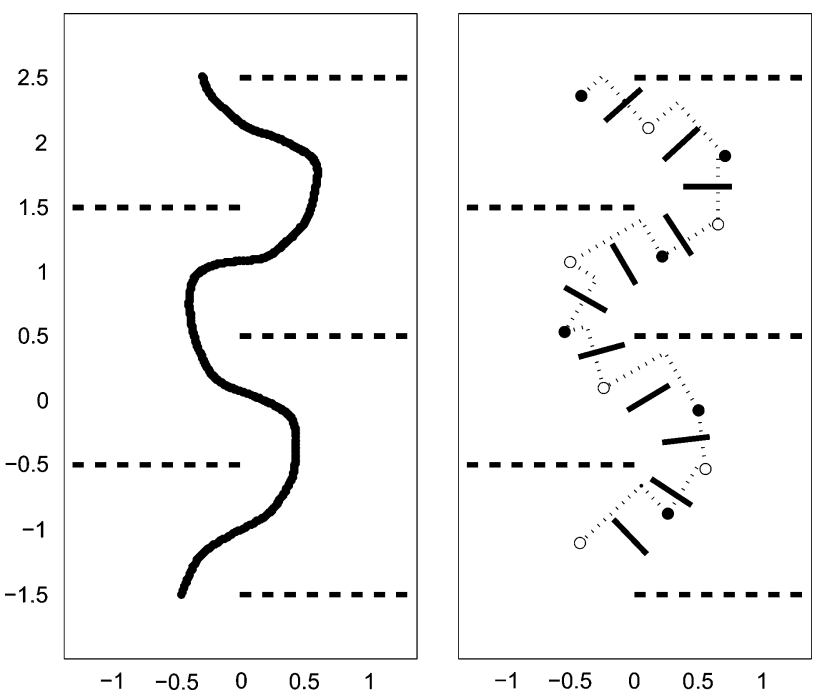

Fig. 2 Dependent measures for a representative trial with dashed lines indicating walls. Left bold line represents the hip trajectory. From this, pathlength and mean speed were derived. Right black and white circles indicate right and left foot positions, respectively. Black line segments represent trunk orientation at each heel strike. Dotted lines indicate step length and width based on this orientation

regression equations based on anthropometric models (provided by Xsens). Second, a calibration procedure was performed during which matching took place between the orientation of a sensor in the global frame and the known orientation of each segment in the neutral $(\mathrm{N})$ pose. This pose is characterized by standing upright and faced forward with the shoulders above hip, hip above knees, and knees above feet. The feet were placed parallel, one foot width apart. Arms extended besides body (vertically) with thumbs forward.

During each trial, participants wore one of the pairs of goggles and were instructed to walk at a comfortable, selfpreferred pace from the start to end position while avoiding contact with the walls. All 16 trials were performed consecutively without interruption.

\section{Dependent measures}

Matlab (Guide 1998) was used to analyze the 3D positional data of 23 body segments produced by the inertial motion capture system. The data were filtered using a low-pass second-order Butterworth filter with a cutoff frequency of $6 \mathrm{~Hz}$. For each trial, several kinematic measures were extracted. First, pathlength was defined as the distance travelled (in $\mathrm{mm}$ ) by the pelvis in the transverse plane between passing the first and last walls. Second, mean speed was defined as the length of the path divided by the temporal interval that elapsed between these two moments of passing. In order to deal with differences in preferred speed, this was then normalized to leg length $L$, defined as 
the vertical displacement of the hip during upright stance (as given by the MVN system). Third, proportional step width was defined as the component of the step that is perpendicular to the direction of movement at the moment of heel strike. This was divided by leg length $L$. Furthermore, the orientation of the trunk at the moment of heel strike was used to define the direction of movement. Mean step width was then calculated per trial as the average proportional step width over all steps within the aforementioned interval. See Fig. 2 for a graphical representation of the dependent measures.

Finally, the total magnitude of head rotation and its mean angular velocity were analyzed using the position of both optical markers. This was done separately for both the transverse (yaw) and the sagittal planes (pitch). The temporal interval over which to calculate these was defined by the moments of passing the first and fourth walls. We decided on this interval to exclude the downward pitch typically observed at the end of each trial (done to see the "finish line" taped down on the floor).

\section{Statistical analysis}

A four (visual field size) $\times$ three (repeated measures) ANOVA was performed for mean speed, pathlength and mean step width. Additionally, a two (rotational direction) $\times$ four (visual field size) $\times$ three (repeated measures) ANOVA was performed for total magnitude of head rotation and head mean angular speed.

Whenever Mauchley's test indicated a violation of the sphericity assumption, a Greenhouse-Geisser correction was applied to the variance analysis as well as a Bonferroni adjustment (instead of Tukey HSD) on the pairwise comparisons (Field 2009). All analyses were performed with STATISTICA 8.0 (2007), and significance levels for each were set to $5 \%$.

\section{Results}

\section{Pathlength}

Visual field size affects pathlength, $F(3,15)=56.650$, $p<.001$. A decrease in visual field size yields an increase in pathlength. Pairwise comparison shows significant differences between all visual field conditions except medium and large (see Fig. 3).

\section{Mean speed}

Visual field size affects the speed of movement, $F(3,15)=$ $38.405 ; p<.001$. A decrease in visual field size leads to a decrease in mean speed. Pairwise comparison shows significant differences between the smallest visual field and all others (see Fig. 4).

Mean step width

Mean step width is affected by visual field size, $F(3$, $15)=3.955 ; p=.029$. Decreasing the size of the visual field yields increased step width. Pairwise comparison shows a significant difference between the smallest and unrestricted visual fields (see Fig. 5).

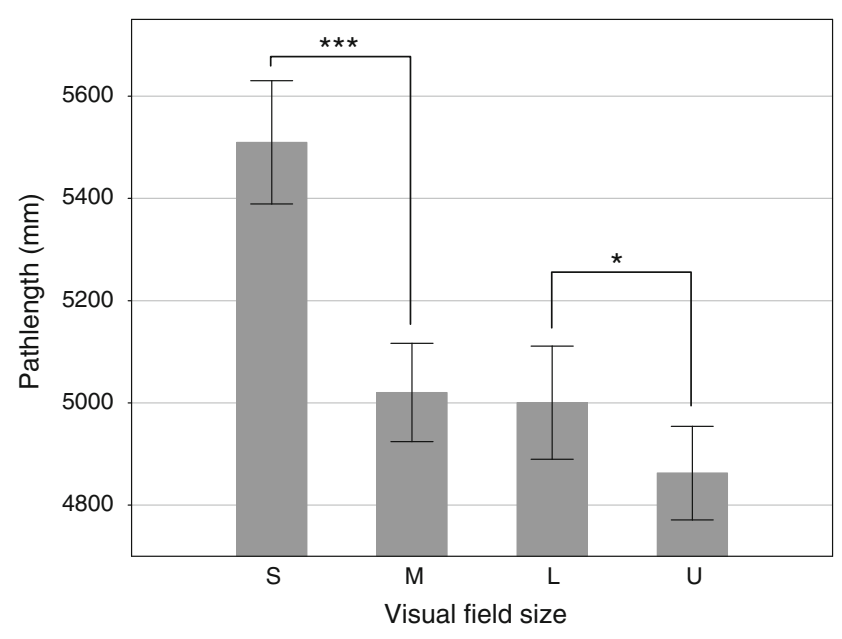

Fig. 3 Pathlength of the hip as a function of visual field size: small $\left(\mathrm{S}: 40^{\circ} \times 25^{\circ}\right)$, medium $\left(\mathrm{M}: 80^{\circ} \times 60^{\circ}\right)$, large $\left(\mathrm{L}: 115^{\circ} \times 90^{\circ}\right)$, and Unrestricted $(\mathrm{U})$. The closest neighboring significantly different pairs are indicated by $*(p<.05), * *(p<.01)$ and $* * *(p<.001)$. Error bars represent standard error

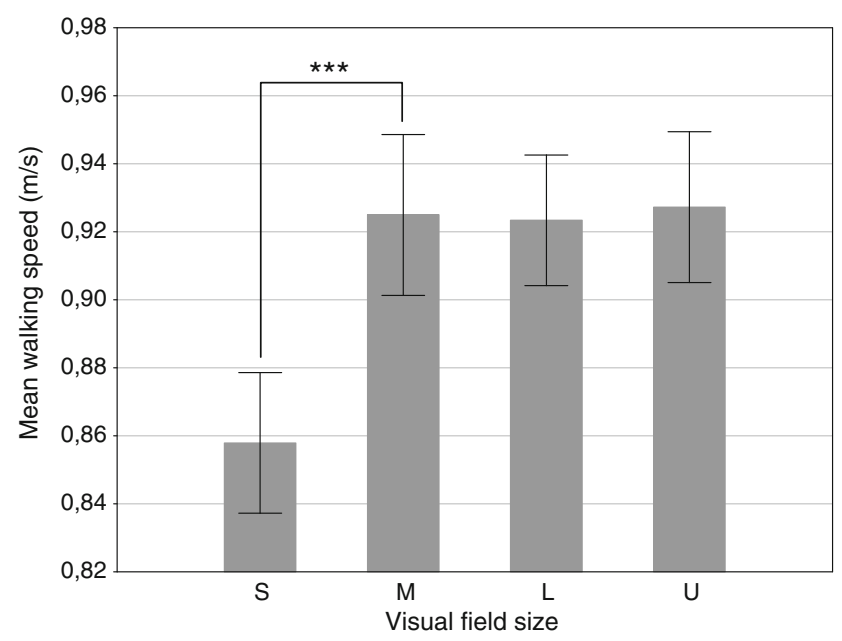

Fig. 4 Mean walking speed over the interval between passing the first and last walls as a function of visual field size: Small (S: $\left.40^{\circ} \times 25^{\circ}\right)$, medium $\left(\mathrm{M}: 80^{\circ} \times 60^{\circ}\right)$, large $\left(\mathrm{L}: 115^{\circ} \times 90^{\circ}\right)$, and unrestricted $(\mathrm{U})$. The closest neighboring significantly different pairs are indicated by $*(p<.05), * *(p<.01)$ and $* * *(p<.001)$. Error bars represent standard error 


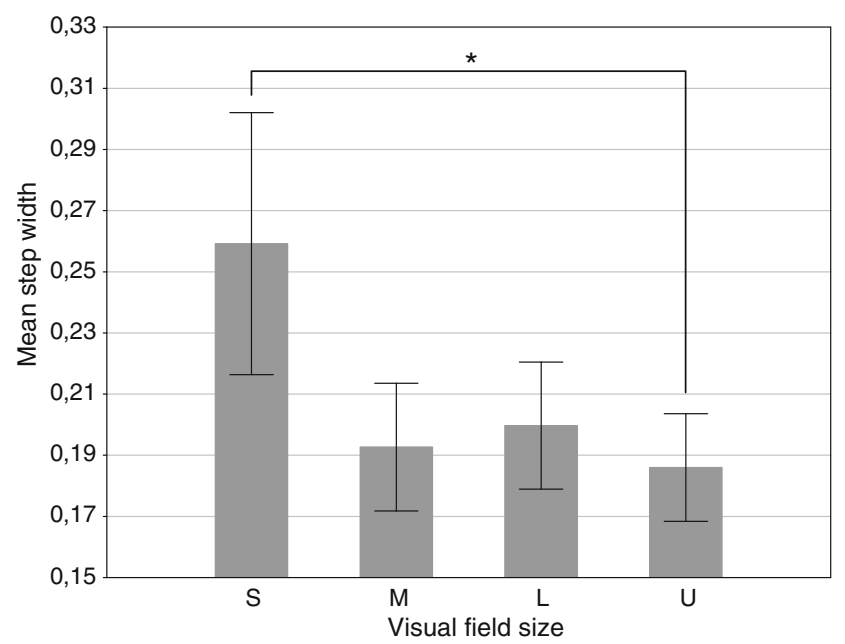

Fig. 5 Mean step width (proportional to leg length $L$ ) as a function of visual field size: small $\left(\mathrm{S}: 40^{\circ} \times 25^{\circ}\right)$, medium (M: $\left.80^{\circ} \times 60^{\circ}\right)$, large (L: $115^{\circ} \times 90^{\circ}$ ), and unrestricted $(\mathrm{U})$. The closest neighboring significantly different pairs are indicated by $*(p<.05), * *(p<.01)$ and $* * *(p<.001)$. Error bars represent standard error
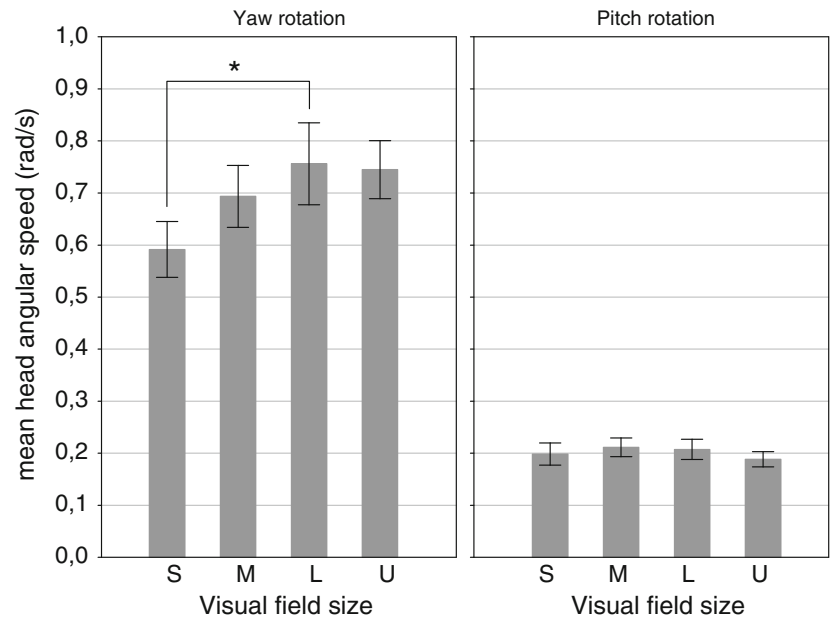

Fig. 6 Head mean angular speed in the yaw (left panel) and pitch (right panel) direction as a function of visual field size: small (S: $40^{\circ} \times 25^{\circ}$ ), medium $\left(\mathrm{M}: 80^{\circ} \times 60^{\circ}\right)$, large $\left(\mathrm{L}: 115^{\circ} \times 90^{\circ}\right)$, and unrestricted $(\mathrm{U})$. The closest neighboring significantly different pairs are indicated by $*(p<.05), * *(p<.01)$ and $* * *(p<.001)$. Error bars represent standard error

\section{Head movement}

Total magnitude of head rotation was greater for yaw than pitch rotation, $F(1,8)=62.950 ; p<.001$. However, this did not vary as a function of visual field size, $F(3,24)=$ $0.227 ; p=.88$.

Yaw mean angular speed was higher than pitch mean angular speed $F(1,8)=89.379 ; p<.001$. Also, a significant interaction effect was found for visual field size $\times$ rotational direction, $F(3,24)=4.299 ; p=.014$. The size of the field affects head mean angular speed in the yaw, but not the pitch direction. Pairwise comparison shows a significant difference in yaw rotation between the smallest and both the large and unrestricted field sizes (see Fig. 6).

\section{Additional results}

In order to investigate whether time-related factors such as practice, fatigue, or boredom have any effect on locomotion through a multiple obstacle environment, five additional analyses were performed. A four (block) $\times$ three (trials) ANOVA was performed for each of the dependent measures. None of these indicated an effect (either main or interaction) of time on steering behavior.

\section{Discussion}

The current study investigated the influence of visual field size on a steering task involving multiple obstacles. The results indicate that restriction of the visual field affects performance. When we take the mean speed and pathlength in the unrestricted condition as a baseline, it is observed that participants move at their desired speed over a path providing them with clearance to the obstacles that permits only small deviation from the planned path. It seems that under these conditions, the perceived threat to safety is minimal and therefore behavior is governed by energy conservative and time efficient strategies. Next, when the visual field is restricted to a medium-large field size (i.e., $80^{\circ} \times 60^{\circ}$ and $115^{\circ} \times 90^{\circ}$ ), we observed that participants enlarged their safety margin by taking a path that increased their clearance around the obstacles. However, they did not slow down. Finally, when confronted with the smallest visual field (i.e., $40^{\circ} \times 25^{\circ}$ ), participants did slow down, next to an additional increase in the pathlength. These observations are in accordance with findings from a recent obstacle crossing study (Jansen et al. 2011), in which we report increased toe clearance when stepping over an obstacle with an intermediate vertical viewing angle (i.e., $40^{\circ}-90^{\circ}$ ). With a small angle of $25^{\circ}$, we observed a further increase in clearance as well as decreased speed of movement. As a consequence of the perceived threat to safety, induced by visual field restriction, all participants chose to optimize safety (collision avoidance) at the cost of spending more energy.

We hypothesized that the reduction in speed of movement observed as a consequence of a small visual field might be the result of additional time needed to execute larger but slower head movements. In order to investigate whether this might be the case here, we analyzed the total magnitude of head rotation as well as the mean angular speed of the head. The results indicate no effect of visual 
field size on the total magnitude of head rotation. However, we did observe a decrease in yaw rotation speed for the smallest visual field condition. This decrease in mean rotational speed of the head as a function of reduced visual field is in line with previous work by Wells and Venturino (1990).

When combining these results with the observed effect of visual field size on walking speed, it seems plausible that participants reduce their walking speed in the $\mathrm{S}$ condition in order to execute the slower head movements that are required by such a small field.

An alternative explanation for this reduced speed of movement might be that it increases the amount of time to prepare for and execute the bodily movement required to avoid the obstacle. Also, in case of a collision, the impact would be minimized.

An important consequence of visual field restriction is the impairment of balance maintenance (Paulus et al. 1984; Turano et al. 1993), which has been shown to affect step width (Gabell and Nayak 1984). The present results show that the average step width increased from approximately $0.19 L$ in the $\mathrm{M}, \mathrm{L}$, and $\mathrm{U}$ conditions to $0.26 \mathrm{~L}$ in the $\mathrm{S}$ condition, indicating balancing problems. The difference between the mean step width found in the unrestricted condition here and the $0.12 L$ reported by Bauby and Kuo (2000) is likely to be explained by the difference between both tasks. They investigated step width during unobstructed walking in a straight line, whereas the task in the present study was to steer through a multiple obstacle environment. The latter requires a constant change in COM direction, which is accompanied by foot placements that enable this redirection. This inevitably enlarges the average step width.

By increasing the width of a step, the base of support becomes wider, preventing increased postural sway to result in a fall. However, this will result in additional energy expenditure (Donelan et al. 2001). Based on the results, we propose that only for the smallest visual field were balance problems substantial enough to warrant this extra cost. Additionally, it seems that the increased length of the travel path as observed in both the $\mathrm{M}$ and $\mathrm{L}$ conditions cannot be accounted for by balancing problems.

Finally, no differences were found between the $\mathrm{M}$ and $\mathrm{L}$ conditions for each of the dependent variables. This similarity in performance is in accordance with previous studies on obstacle avoidance behavior under restricted viewing conditions, which reported a similar performance plateau (Toet et al. 2008; Jansen et al. 2011).

It should be noted that the walls used in this experiment do not simulate a closed indoor environment, since they were constructed as open frames to permit tracking of the optical markers. This means that participants could always see the remaining obstacles as well as the goal position.
In addition, it should be mentioned that the obstacles were evenly spaced throughout the environment, which does not simulate any specific real world situation. It would be interesting to see if similar behavioral patterns emerge when using opaque walls placed at varying distances.

To summarize the results: we investigated how visual field size affects steering behavior through a multiple obstacle environment. The results suggest that compared with an unrestricted visual field, an intermediate field size causes participants to select a wider path around the obstacles without slowing down or altering step width. Alternatively, when confronted with a small visual field (i.e., $40^{\circ} \times 25^{\circ}$ ), participants did slow down and increased their step width in addition to further enlarging their obstacle clearance. Therefore, we conclude that for all visual field limitations, participants chose to optimize safety (collision avoidance) at the cost of spending more energy. However, it seems that only for the smallest viewing condition, safety concerns were substantial enough to warrant the additional metabolic cost associated with increased step width. This precaution may be taken in order to deal with the balance impairment caused by the extensive lack of input from the peripheral visual field. Moreover, we suggest that this change in locomotion characteristics may well be the result of a transition from an energy conservative and time efficient strategy to one that emphasizes safety. In addition, it may be that the reduction in speed of movement observed as a consequence of a small visual field is the result of additional time needed to execute a similar magnitude of head rotation, but at a lower speed.

The results of this study give insight into human strategy changes during a steering task with a limited visual field. Several areas may benefit from such insight. First, it adds to the understanding of how people confronted with a limited visual field move through structured environments. Sometimes this is the result of eye diseases such as retinitis pigmentosa and glaucoma. There, it is useful to know how adaptive motor behavior is affected in clinical eye patients. On the other hand, specific hardware (e.g. HMDs) affects visual field size by choice of display size and positioning, which has a trade-off in optical complexity, and therefore costs. These choices may benefit from the present results by formulating guidelines for the selection and development of such devices. Moreover, by investigating the (changes in) strategies associated with steering under restricted viewing conditions, we contribute to the understanding of human adaptive locomotion, especially under suboptimal conditions. In this regard, it would be very interesting to see if similar strategy changes can be found during other perceptuomotor tasks and under other circumstances. Examples of the latter could be diminished lighting conditions and irregular surfaces. 
Acknowledgments This research has been supported by the GATE project, funded by the Netherlands. Organization for Scientific Research (NWO) and the Netherlands ICT Research and Innovation Authority (ICTRegie).

Open Access This article is distributed under the terms of the Creative Commons Attribution Noncommercial License which permits any noncommercial use, distribution, and reproduction in any medium, provided the original author(s) and source are credited.

\section{References}

Bauby C, Kuo A (2000) Active control of lateral balance in human walking. J Biomech 33:1433-1440

Chapman G, Hollands M (2007) Evidence that older adult fallers prioritise the planning of future stepping actions over the accurate execution of ongoing steps during complex locomotor tasks. Gait Posture 26:59-67

Courtine G, Schieppati M (2003) Human walking along a curved path. I. Body trajectory, segment orientation and the effect of vision. Eur J Neurosci 18:177-190

Creem-Regehr SH, Willemsen P, Gooch AA, Thompson WB (2005) The influence of restricted viewing conditions on egocentric distance perception: implications for real and virtual environments. Perception 34:191-204

Donelan J, Kram R, Arthur D (2001) Mechanical and metabolic determinants of the preferred step width in human walking. Proc R Soc Lond B Biol Sci 268:1985-1992

Donelan J, Shipman D, Kram R, Kuo A (2004) Mechanical and metabolic requirements for active lateral stabilization in human walking. J Biomech 37:827-835

Field A (2009) Discovering statistics using SPSS. SAGE publications Ltd

Gabell A, Nayak U (1984) The effect of age on variability in gait. J Gerontol 39:662-666

Guide M (1998) The MathWorks Inc., Natick

He Z, Wu B, Ooi T, Yarbrough G, Wu J (2004) Judging egocentric distance on the ground: occlusion and surface integration. Perception 33:789-806

Hollands M, Sorensen K, Patla A (2001) Effects of head immobilization on the coordination and control of head and body reorientation and translation during steering. Exp Brain Res 140:223-233

Hollands M, Patla A, Vickers J (2002) "Look where you're going!": gaze behaviour associated with maintaining and changing the direction of locomotion. Exp Brain Res 143:221-230

Inman V (1966) Human locomotion. Can Med Assoc J 94:1047-1054

Jansen S, Toet A, Delleman N (2010) Restricting the vertical and horizontal extent of the field-of-view: effects on manoeuvring performance. Ergon Open J 3:19-24

Jansen S, Toet A, Werkhoven P (2011) Obstacle crossing with lower visual field restriction: shifts in strategy. J Mot Behav 43:55-62

Marigold D (2008) Role of peripheral visual cues in online visual guidance of locomotion. Exerc Sport Sci Rev 36:145-151

Menz H, Lord S, Fitzpatrick R (2003) Age-related differences in walking stability. Age Ageing 32:137-142

Mohagheghi A, Moraes R, Patla A (2004) The effects of distant and on-line visual information on the control of approach phase and step over an obstacle during locomotion. Exp Brain Res 155:459-468

Paquette M, Vallis L (2010) Age-related kinematic changes in late visual-cueing during obstacle circumvention. Exp Brain Res 203:1-12
Paquette M, Fuller J, Adkin A, Vallis L (2008) Age-related modifications in steering behaviour: effects of base-of-support constraints at the turn point. Exp Brain Res 190:1-9

Patla A, Vickers J (1997) Where and when do we look as we approach and step over an obstacle in the travel path? Neuroreport $8: 3661-3665$

Patla A, Adkin A, Ballard T (1999a) Online steering: coordination and control of body center of mass, head and body reorientation. Exp Brain Res 129:629-634

Patla A, Prentice S, Rietdyk S, Allard F, Martin C (1999b) What guides the selection of alternate foot placement during locomotion in humans. Exp Brain Res 128:441-450

Paulus W, Straube A, Brandt T (1984) Visual stabilization of posture. Physiological stimulus characteristics and clinical aspects. Brain J Neurol 107:1143-1163

Prévost P, Yuri I, Renato G, Alain B (2003) Spatial invariance in anticipatory orienting behaviour during human navigation. Neurosci Lett 339:243-247

Roetenberg D, Luinge H, Slycke P (2009) Xsens MVN: full 6DOF human motion tracking using miniature inertial sensors. In: Xsens Motion Technologies BV, Tech. Rep

Rushton S, Harris J, Lloyd M, Wann J (1998) Guidance of locomotion on foot uses perceived target location rather than optic flow. Curr Biol 8:1191-1194

Saunders J (1953) The major determinants in normal and pathological gait. J Bone Joint Surg 35:543-558

Szoboszlay Z, Haworth L, Reynolds T, Lee A, Halmos Z (1995) Effect of field-of-view restriction on rotocraft pilot workload and performance: preliminary results. In, SPIE, p 142

Toet A, Jansen S, Delleman N (2008) Effects of field-of-view restriction on manoeuvring in a 3-D environment. Ergonomics 51:385-394

Turano K, Herdman S, Dagnelie G (1993) Visual stabilization of posture in retinitis pigmentosa and in artificially restricted visual fields. Invest Ophthalmol Vis Sci 34:3004-3010

Turano K, Rubin G, Quigley H (1999) Mobility performance in glaucoma. Invest Ophthalmol Vis Sci 40:2803-2809

Vallis L, McFadyen B (2003) Locomotor adjustments for circumvention of an obstacle in the travel path. Exp Brain Res 152:409-414

Vargas-Martin F (2002) Augmented-view for restricted visual field: multiple device implementations. Optom Vis Scince 79:715-723

Warren W, Blackwell A, Kurtz K, Hatsopoulos N, Kalish M (1991) On the sufficiency of the velocity field for perception of heading. Biol Cybern 65:311-320

Warren W, Kay B, Zosh W, Duchon A, Sahuc S (2001) Optic flow is used to control human walking. Nature Neurosci 4:213-216

Weiß C (2007) StatSoft, Inc., Tulsa, OK.: STATISTICA, version 8. AStA Adv Stat Anal 91:339-341

Wells M, Venturino M (1990) Performance and head movements using a helmet-mounted display with different sized fields-ofview. Opt Eng 29:870-877

Werner E, Rossi C (1991) Manual of visual fields. Churchill Livingstone, NewYork

WorldViz P (2005) 3D optical tracker

Wu B, Ooi T, He Z (2004) Perceiving distance accurately by a directional process of integrating ground information. Nature 428:73-77

Zarrugh M, Todd F, Ralston H (1974) Optimization of energy expenditure during level walking. Eur J Appl Physiol 33:293-306 